\title{
An Unusual Case of Migraine Preconception and Severe Postpartum Headache and Intracranial Hemorrhage
}

\author{
Nighat Fatima Ayesha Salman Lama Khalid Hamza Tooba Shakeel \\ Department of Obstetrics and Gynecology, Dubai Hospital, Dubai Health Authority, Dubai, UAE
}

\section{Keywords}

Migraine $\cdot$ Pregnancy $\cdot$ Venous thromboembolism ·

Ischemic strokes

\begin{abstract}
Migraine affects many women in their childbearing years and gets worse during pregnancy, probably due to fluctuations in reproductive hormones. Similar changes in maternal physiology due to pregnancy increase the susceptibility toward secondary headache disorders and can cause the return of migraines in the postpartum period.

Increased occurrence of headaches is also associated with the administration of epidural anesthesia, which decreases cerebrospinal fluid pressure. However, the less common causes such as secondary headache disorders related to cerebrovascular disorders and hypertension should be considered for differential diagnosis in this group of patients. In this case report, we discuss the case history of a 36-yearold woman, with a past history of migraine without aura, who presented at 1 week postpartum with headache, confusion, dizziness, right-sided weakness, and urinary incontinence. Brain imaging studies revealed global hypoxic ischemic injury to the brain. This case report discusses her presentation, investigations, management, and etiology of migraine, leading to stroke and death postpartum.
\end{abstract}

(c) 2020 The Author(s).

Published by S. Karger AG, Basel karger@karger.com www.karger.com/dmj

Karger $\stackrel{\text { ' }}{5}$

BOPEN ACCESS
(C) 2020 The Author(s)

Published by S. Karger AG, Basel

This article is licensed under the Creative Commons AttributionNonCommercial-NoDerivatives 4.0 International License (CC BYNC-ND) (http://www.karger.com/Services/OpenAccessLicense). Usage and distribution for commercial purposes as well as any distribution of modified material requires written permission.

\section{Introduction}

Migraine, or recurrent headache, is among the most common nervous disorders that result due to escalating excitability of the central nervous system. It can be extremely painful and disabling, associated independently or in combination with neurological, autonomic, and gastrointestinal alteration $[1,2]$. Two primary forms of migraines have been distinguished: migraine without aura (MO) and migraine with aura (MA) [3]. MO is a characteristic migraine experienced by most individuals suffering from migraines and includes severe pain on 1 or both sides of the head associated with nausea, vomiting, and sensitivity to light or sound. On the other hand, MA is the classic migraine that usually causes visual disturbances along with other migraine symptoms.

Women are more prone to develop migraine, with an incidence of $18 \%$ compared to $6 \%$ incidence among men [4]. This rise in the occurrence of migraine in women occurs in their fertile/childbearing years. Women with migraine have greater risks of pregnancy-associated hypertension disorders, miscarriage, low birth weight, and preterm birth than women without migraine. Balancing the risks and benefits of migraine treatment against the potential risks to the mother and fetus can be challenging, warranting an individualized migraine treatment plan [5].

Migraine often improves spontaneously during the second or third trimester of pregnancy but may also 
worsen. The reduction in migraine attacks during pregnancy can be attributed to the increased levels of estrogen and endogenous opioid that raise the threshold of pain and prevent hormonal fluctuations [4]. After childbirth, migraine relapse may occur, especially in the initial month, probably due to the fall in the estrogen level, postpartum depression, or new parental roles [6].

In the present study, we refer to a case of a 36-year-old woman with a history of MO who presented with postpartum headache, confusion, dizziness, right-sided weakness, and urinary incontinence 1 week after childbirth. Brain imaging studies revealed global hypoxic ischemic injury to the brain. This case report discusses her presentation, investigations, management, and etiology of migraine preconception that led to stroke postpartum, subsequently resulting in her death.

\section{Case Study}

\section{Case Presentation}

A 36-year-old woman, gravida 3 para 3, who delivered vaginally at 37 weeks gestation presented with headaches, dizziness, and vomiting associated with right-sided weakness 7 days post-delivery. On examination, she was found to be drowsy and her speech was incoherent.

\section{Past Medical History}

The patient had a medical history of migraine for 1 year, for which she had visited a neurologist and was given symptomatic treatment. Antenatally, her migraine had settled, and pregnancy remained uneventful. She had received intrapartum epidural anesthesia.

\section{Postpartum Symptoms}

Post-delivery she was kept as in-patient due to headache, vomiting, and confusion; however, there were no signs of systemic illness.

\section{Clinical Assessment, Differential Diagnosis, and Management}

On examination, her blood pressure was normal without preeclampsia. Moreover, blood glucose level and ECG were also found to be normal. A differential diagnosis of postpartum mental health illness and post-dural puncture headache were made, and treatment was initiated accordingly. Since her symptoms were persistent, she was reviewed by a neurologist.

A differential diagnosis of cerebrovascular accident was made; however, cerebrovascular events including in- tracerebral hemorrhage (ICH) arising in pregnancy or peripartum are often due to new onset preeclampsia and eclampsia, especially when acute blood pressure is not controlled, but in this case she was normotensive. She was vitally stable, oriented but confused, and her Glasgow Coma Scale (GCS) was 14/15. Her right fist was clenched with a right pronator drift and restricted movement on her right side as compared to the left side.

Urgent computed tomography (CT) scan revealed acute cerebral hemorrhage, since she was on thromboprophylaxis after her delivery as per venous thromboprophylaxis risk assessment score; after the CT scan report, her treatment was withheld and the patient was shifted to the intensive care unit (ICU) for further management. Other differential diagnosis was cerebral venous thrombosis (CVT); although a CT angiography ruled out CVT, early filling vascular structures were observed from the left middle cerebral artery. Therefore, a brain magnetic resonance imaging was scheduled.

In the ICU, the patient was managed by a multidisciplinary team, and she remined vitally stable, conscious with GCS15/15 with right-sided weakness $4 / 5$, which improved by physiotherapy. She maintained her bowel and urinary functions.

So, the neurosurgeons decided for conservative management without any surgical intervention. Repeated CT scan (third day) revealed same findings with no interval changings. No midline defect or acute infarction or hemorrhage was detected.

In view of recovering physical activity, the patient was transferred from the ICU to high dependency unit after 5 days. After 2 days, her condition suddenly deteriorated, and she developed signs of pulmonary embolism. She became tachypneic, tachycardiac, and desaturated; therefore, immediate intubation was carried out and urgent CT pulmonary angiogram (CTPA) with brain CT scan was performed. The CTPA showed extensive pulmonary thromboembolism with significant right ventricular strain, whereas brain $\mathrm{CT}$ showed a resolving $\mathrm{ICH}$.

A multidisciplinary team decided for embolectomy; however, she developed cardiac arrest. Cardiopulmonary resuscitation was performed successfully and percutaneous thrombectomy for bilateral pulmonary embolism was done. An inferior vena cava filter was placed in the infrarenal inferior vena cava.

Heparin infusion was given, but thrombolytic agents were avoided in view of ICH. Follow-up brain CT 2 days later revealed diffuse brain swelling and coning, highly suggestive of global hypoxic ischemic injury to the brain. Neurosurgical assessment revealed absence of brain stem 


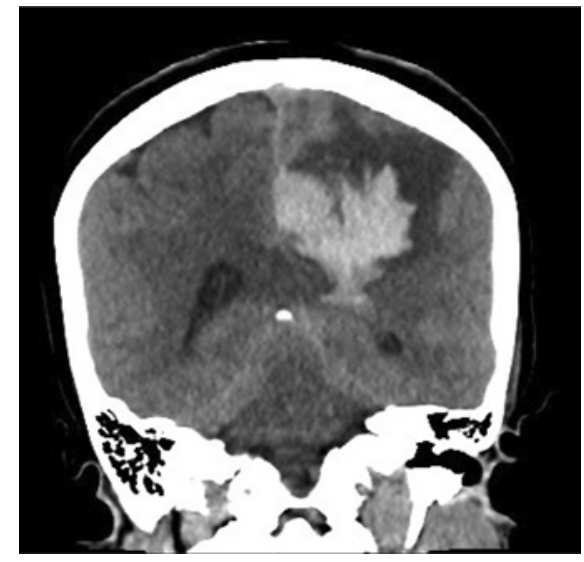

Fig. 1. CT brain with irregular hematoma in the left posterior, frontal, and parietal regions with extension to both lateral and third ventricles (suggestive of subarachnoid hemorrhage). CT, computed tomography.

reflexes. The patient developed cardiac arrest again; she could not be revived despite being given advanced cardiopulmonary resuscitation and was declared dead.

\section{Investigations}

Brain CT scan revealed a large, irregular-shaped heterogenous intraparenchymal acute hematoma in the left posterior frontal and parietal regions, measuring at least $4.7 \times 4.8 \times 4.0 \mathrm{~cm}$. Further, hyper-density was noted in both the lateral ventricles, more in the left ventricle than in the right ventricle. An extension of the intraventricular hemorrhage was observed in the third ventricle, suggesting an acute subarachnoid hemorrhage (Fig. 1 below).

The CT angiogram neither showed any obvious filling defect in the opacified major dural venous sinuses nor any obvious aneurysmal dilatation or gross major vascular malformation in the visualized portions of anterior and posterior circulation. Acute pulmonary thromboembolism with significant right ventricular strain was revealed by CTPA.

A follow-up brain CT scan showed no significant interval change in the size of the previously visualized left parietal lobe hematoma. In the last CT scan, the following developments were noted that suggested global hypoxic ischemic injury to the brain: subarachnoid hemorrhages in the bilateral cerebral convexities, interval development of diffuse cerebral edemas with loss of gray-white differentiation, and diffuse brain swelling with effacement of cerebral cortical sulci, cisternal spaces, and compression of both lateral ventricles.

\section{Differential Diagnosis}

From our clinical assessment and patient's medical history, a differential diagnosis of post-dural puncture headache and ICH and CVT was made.

\section{Discussion}

This is an unusual case of a young patient with migraine preconception followed by postpartum headache and ICH leading to death. A remarkable number of women experience pregnancy-related stroke that can lead to increased morbidity and mortality.

For an impartial diagnosis of headaches, it is necessary to collect information about the history of the condition. Also, patient details before pregnancy is an important predictor of development of migraine during or after pregnancy [6].

The management of postpartum headaches/migraine is dependent on the source of the headache. The common causes are mostly exacerbation of the primary headache syndromes, such as migraine or tension headache. However, postpartum headaches could be due to life-threatening secondary causes, such as primary intracranial pathology or obstetric complications [7]. Hence, these secondary causes should be considered while managing a case of postpartum headache.

It has long been suggested that headache and migraine may be precursors of stroke, in particular ischemic stroke [8]. A large amount of evidence has been published linking MA to stroke and other cardiovascular events, including several meta-analyses examining the actual risk of ischemic stroke in people with migraine. They all suggest the overall risk of stroke is higher in people with migraine than in those without migraine. Two studies found that the risk of hemorrhagic stroke was higher in women with migraine than in men with migraine.

Thrombophilia or hypercoagulability is commonly associated with migraine and pregnancy. Notably, the probability of stroke is the highest in the postpartum period. In the first few days postpartum, the risk of subarachnoid hemorrhage is high. Cerebrovascular thrombosis-related headache is mostly diffused, progressive, and severe but can also be unilateral, sudden, and similar to migraine. Thus, when a young, pregnant, or postpartum woman presents with headaches or seizures, cerebrovascular thrombosis risk factors should be considered and investigated [9]. Although with a number of controversial treatment options, heparin has been considered safe and effective even when cerebrovascular thrombosis is accompanied by hemorrhagic lesions. 
There are case reports of intracranial hemorrhage in pregnancy or soon after delivery, and the outcome mainly depends upon timely recognition and intervention, but all patients will have neurological deficit $[10,11]$. In conclusion, hypercoagulability is common both in migraine and pregnancy. Hence, cerebrovascular disorders such as venous thromboembolism and ischemic or hemorrhagic strokes demand attention for timely management in order to reduce complications [4].

\section{Learning Points/Take Home Messages}

Recognize the importance of multidisciplinary input in the management of pregnant women with history of migraine and prompt investigations of severe postpartum headache. Imaging should be done promptly to allow for early treatment and optimize outcomes.

\section{Statement of Ethics}

Patient guardian (husband) has given written informed consent and has given the permission to publish the case.

\section{Conflict of Interest Statement}

All authors have no conflict of interest.

\section{Author Contributions}

N.F. is the primary author; A.S., L.K., and T.S. are coauthors. All authors have contributed in helping to format and follow the case. All authors have no conflict of interest.

\section{References}

1 Skajaa N, Szépligeti SK, Xue F, Sørensen HT, Ehrenstein V, Eisele O, et al. Pregnancy, birth, neonatal, and postnatal neurological outcomes after pregnancy with migraine. Headache. 2019;59(6):869-79.

2 WHO. Headache disorders. Available from: https://www.who.int/news-room/fact-sheets/ detail/headache-disorders. 2016.

3 Arnold M. Headache classification committee of the international headache society (IHS) the international classification of headache disorders. Cephalalgia. 2018;38(1):1211.

4 Allais G, Chiarle G, Sinigaglia S, Mana O, Benedetto C. Migraine during pregnancy and in the puerperium. Neurol Sci. 2019;40(Suppl 1):81-91.
5 Frederick IO, Qiu C, Enquobahrie DA, Aurora SK, Peterlin BL, Gelaye B, et al. Lifetime prevalence and correlates of migraine among women in a pacific northwest pregnancy cohort study. Headache. 2014;54(4): 675-85.

6 Negro A, Delaruelle Z, Ivanova TA, Khan S, Ornello R, Raffaelli B, et al. Headache and pregnancy: a systematic review. J Headache Pain. 2017;18(1):106.

7 Boushra M, Rathbun KM. Postpartum headache. Treasure Island, FL: StatPearls Publishing LLC; 2019.

8 Lantz M, Kostulas K, Waldenlind E, Sjöstrand C. Prevalence of migraine headache in an inpatient stroke population. Acta Neurol Scand. 2015;131(5):290-7.
9 Han KH, Won YD, Na MK, Han MH, Ryu JI, Kim JM, et al. Postpartum superior sagittal sinus thrombosis: a case report. Korean J Neurotrauma. 2018;14(2):146-9.

10 Stein-Fredbeck L, Rosenberg R, Frank R. A case report of maternal cerebral hemorrhage in preterm pregnancy. J Obstet Gynecol Neonatal Nurs. 2017;46(4):609-16

11 Zvyagina L. Intracranial haemorrhage due to post-partum cerebral angiopathy. 2012. Available from: https://www.eanpages. org/2012/11/01/case-report-intracranialhaemorrhage-due-to-post-partum-cerebralangiopathy/. 